\title{
Perfeccionismo e a relação com psicopatologias: Estudo integrativo
}

\author{
Perfectionism and the relationship with psychopathologies: Integrative study \\ El perfeccionismo y la relación con las psicopatologías: Estudio integrador
}

Recebido: 16/02/2021 | Revisado: 21/02/2021 | Aceito: 22/02/2021 | Publicado: 01/03/2021

\author{
André Sousa Rocha \\ ORCID: https://orcid.org/0000-0002-0185-9699 \\ Universidade São Francisco, Brasil \\ E-mail: andresousarocha9@gmail.com
}

\begin{abstract}
Resumo
O perfeccionismo é um traço latente psicológico que vem sendo compreendido de diferentes formas. Os avanços científicos e metodológicos na área sugerem que esse fenômeno possui componentes positivos e negativos. Esse estudo motivou-se pela questão problema levantada: quais as principais psicopatologias, na atualidade, relacionadas ao perfeccionismo? Algumas discussões são promovidas nesse ponto de vista. Para tanto, o presente artigo condensa, organiza e apresenta uma revisão integrativa consultada em bases nacionais e internacionais e visou investigar quais as principais psicopatologias relacionadas ao perfeccionismo. Desse modo, foram executadas buscas de artigos nas bases de dados Medline, Pubmed e Springer com os descritores perfeccionismo, psicopatologia, perfectionism e psychopathology ambos recrutados do DeCS e MeSH. Dos 226 artigos encontrados inicialmente, 213 foram descartados à medida que os critérios de inclusão e exclusão iam sendo aplicados. Desse modo, 13 artigos compuseram a amostra final. Os resultados indicaram que o perfeccionismo tem se relacionado com depressão, ansiedade, transtornos alimentares (bulimia e anorexia) bem como transtorno obsessivo compulsivo. Limitações e sugestões de futuros estudos são discutidos ao final.
\end{abstract}

Palavras-chave: Perfeccionismo; Saúde mental; Transtornos alimentares.

\begin{abstract}
Perfectionism is a latent psychological trait that has been understood in several different ways. Scientific and methodological advances in the area suggest that this phenomenon has both positive and negative components. Motivated by the question raised: what are the main psychopathologies, currently, related to perfectionism? Some discussions are promoted from this point of view. To this end, this article condenses, organizes and presents an integrative review consulted on national and international bases and aimed to investigate which are the main psychopathologies related to perfectionism. Thus, searches for articles were performed in the Medline, Pubmed and Springer databases with the descriptors perfectionism, psychopathology, perfectionism and psychopathology, both recruited from DeCS and MeSH. Of the 226 articles found initially, 213 were discarded as the inclusion and exclusion criteria were applied. Thus, 13 articles made up the final sample. The results indicated that perfectionism has been related to depression, anxiety, eating disorders (bulimia and anorexia) as well as obsessive compulsive disorder. Limitations and suggestions for future studies are discussed at the end.
\end{abstract}

Keywords: Perfectionism; Mental health; Eating disorders.

\section{Resumen}

El perfeccionismo es un rasgo psicológico latente que se ha entendido de varias formas diferentes. Los avances científicos y metodológicos en el área sugieren que este fenómeno tiene componentes tanto positivos como negativos. Motivado por la pregunta planteada: ¿cuáles son las principales psicopatologías, actualmente, relacionadas con el perfeccionismo? Algunas discusiones se promueven desde este punto de vista. Para ello, este artículo condensa, organiza y presenta una revisión integradora consultada a nivel nacional e internacional y orientada a investigar cuáles son las principales psicopatologías relacionadas con el perfeccionismo. Así, se realizaron búsquedas de artículos en las bases de datos Medline, Pubmed y Springer con los descriptores perfeccionismo, psicopatología, perfeccionismo y psicopatología ambos reclutados de DeCS y MeSH. De los 226 artículos encontrados inicialmente, 213 fueron descartados por aplicar los criterios de inclusión y exclusión. Así, 13 artículos conformaron la muestra final. Los resultados indicaron que el perfeccionismo se ha relacionado con la depresión, la ansiedad, los trastornos alimentarios (bulimia y anorexia) y el trastorno obsesivo compulsivo. Las limitaciones y sugerencias para estudios futuros se discuten al final.

Palabras clave: Perfeccionismo; Salud mental; Trastornos de la alimentación. 


\section{Introdução}

O perfeccionismo é um traço latente psicológico que vem sendo compreendido de diversas formas. Os avanços científicos e metodológicos na área sugerem que esse fenômeno possui componentes positivos e negativos (Afshar et al., 2011). Dentre as características negativas, incluem-se esforços para obter a perfeição e estabelecer padrões extremamente altos de desempenho. Além disso, avaliações excessivamente críticas do autocomportamento e do comportamento de outrem são atributos que qualificam esse construto (Frost, Marten, Lahart, \& Rosenblate, 1990; Hewitt \& Flett, 1991; Stoeber, 2018). Em contrapartida, o perfeccionismo pode ser vantajoso em diversas circunstância, especialmente, quando os indivíduos estão engajados e focados em melhorar o rendimento em tarefas orientadas bem como a propensão em definir metas e objetivos na busca do sucesso (Andrews, Bullock-Yowell \& Nicholson, 2014).

$\mathrm{Na}$ literatura, tem-se encontrados descrições do perfeccionismo em normal e adaptativo versus neurótico e desadaptativo (Bieling, Israelense, \& Antony, 2004). O primeiro tem sido considerado uma disposição que os indivíduos apresentam em definir objetivos que atuam como motivadores para modificar uma situação em que há padrões elevados de cobranças. Por esse motivo, existe uma adaptação que influencia na satisfação a partir de uma dada realização. Nesse sentido, a palavra adaptativa faz toda diferença, uma vez que há compreensão de que o estado de perfeição é dificilmente atingindo e que a definição para alcançar metas elevadas tem limites e alcances. O segundo é conceituado como indivíduos inflexíveis na realização de metas, excessivo crítico do próprio comportamento, mentalidade de tudo ou nada, conhecido como distorções cognitivas, procrastinação e desanimo por metas não atingíveis (Andrews et al., 2014; Flett, Hewitt, Shapiro, \& Rayman, 2001; Hamachek, 1978; Haring, Hewitt, \& Flett, 2003; Martin \& Ashby, 2004)

Há também um foco na relação entre perfeccionismo e os traços da personalidade humana. Especificamente, o modelo dos cinco grandes fatores representados pelo acrônimo OCEAN: abertura à experiência, conscienciosidade, extroversão, amabilidade e neuroticismo (Bojanić, Šakan \& Nedeljković, 2018; Fowler, Davis, Both \& Beth, 2018; Smith, Sherry, Didovic, Saklofske, Stoeber \& Benoit, 2019; Stricker, Buecker \& Schneider \& Preckel, 2019). De acordo com os fatores representados nesse modelo, conscienciosidade e neuroticismo parecem ser os mais pertinentes de serem discutidos em relação ao perfeccionismo.

A conscienciosidade representa diferenças na organização, produtividade e responsabilidade. Indivíduos conscienciosos prezam por ordem e trabalham de forma persistente para atingir seus objetivos além de estarem dedicados a cumprir com obrigações e deveres. Em contraponto, indivíduos menos conscienciosos se sentem confortáveis com a falta de organização e menos motivados em executar tarefas (Soto, 2018). Fowler, Vidovic, Sherry, Stewart \& Saklofske (2018). relevaram uma correlação positiva entre perfeccionismo e conscienciosidade, especialmente, com as facetas organização e esforço por excelência. Os resultados obtidos foram de ( $r=0,58$ e $r=0,34$, respectivamente). Portanto, a presente pesquisa supõe que a dimensão da conscienciosidade está associada a aspectos mais adaptativos e menos desadaptativos do perfeccionismo

Enquanto que neuroticismo ou estabilidade emocional compreende diferenças na frequência e intensidade de emoções negativas. Pessoas que pontuam alto em instrumentos que avaliam essa faceta tendem a experimentar com mais frequência sentimentos de depressão, ansiedade, tristeza e alterações no humor, enquanto que pontuações baixas sugerem indivíduos emocionalmente estáveis, portanto apresentando-se maneira calma e resiliente (Costa \& McCrae, 1992; Soto, 2018). Smith et al. (2019) encontraram uma relação entre perfeccionismo e neuroticismo. Os resultados demonstraram correlação positiva $(r=$ 0,37) entre o neuroticismo e a dimensão do perfeccionismo socialmente prescrito e relações de baixa magnitudes, mas positivas com o perfeccionismo auto orientado $(r=0,15)$ e perfeccionismo orientado para o outro $(r=0,14)$.

Esse estudo motivou-se a partir da seguinte questão-problema levantada: quais as principais psicopatologias, na atualidade, relacionadas ao perfeccionismo? Partindo desse pressuposto, o foco principal do manuscrito objetiva examinar na 
literatura especializada, por meio de uma revisão integrativa, em artigos de periódicos internacionais e nacionais, no período de 2016 a 2021, quais são as psicopatologias mais problematizadas.

\section{Metodologia}

O presente manuscrito constitui-se em uma pesquisa de delineamento bibliográfico integrativo visando uma análise oportuna da literatura. A revisão integrativa permite mapear sobre uma determinada área específica, de modo que sejam compilados em um estudo, diversas opiniões de pesquisadores sobre um tema em pauta. Considera-se que esse tipo de revisão é uma forma eficaz de apresentar os avanços em uma área bem como temática relevante e incitar que outros pesquisadores se engajarem e elaborar estratégias eficazes em âmbitos laborais (Cerqueira, Cardoso, Viana \& Lopes, 2018).

Para o desenvolvimento, necessitou-se a elaboração de registros, análise e organização dos dados bibliográficos para assim permitir uma melhor apreensão da temática de modo a possibilitar o surgimento de criticidade sobre o assunto. Os seguintes critérios de inclusão foram concebidos: a) estudos produzidos dentro do período aprazado, isto é, 2016 a 2021; b) publicações em português e inglês; c) textos originais e disponíveis na íntegra. Como critérios de exclusão têm-se: d) textos não revisados por pares; e) teses e dissertações provenientes de programas de pós-graduação; f) artigos que não versem diretamente sobre a temática abordada; g) artigos que relacionassem perfeccionismo e psicopatologias a alguma abordagem psicológica específica (e.g. Análise do Comportamento e Teoria Cognitivo-Comportamental)

Inicialmente, a partir dos critérios de inclusão e exclusão determinados, foi executada a leitura dos títulos, recrutados a partir dos descritores selecionados; em seguida, leu-se os resumos dos artigos nos quais os títulos faziam menção aos objetivos investigativos, para que assim fosse possível selecionar os artigos de acordo com a área do conhecimento. Consecutivamente, os artigos foram lidos na íntegra e selecionados conforme os critérios da pesquisa.

Foi realizado um recorte temporal nas publicações nos últimos cinco anos, ou seja, 2016 a 2021, por se mostrarem atualizadas no campo científico. Os artigos incluídos deveriam estar contidos nas seguintes bases de dados: Medical Literature Analysis and Retrieval System Online (Medline), PubMed e Springer. Os descritores "perfeccionismo AND psicopatologias" e perfectionism AND psychopathology, foram extraídos conforme os Descritores em Ciências da Saúde (DeCS) com abrangência latino-americano e sul-americano. Ou seja, a tentativa foi resgatar textos na língua brasileira e por meio do Medical Subject Headings - MeSH para recuperar textos produzidos em diferentes localidades. A figura 1 ilustra detalhadamente o processo de busca dos artigos conforme as bases de dados preconizadas.

Figura 1: Fluxograma da identificação e seleção dos artigos para revisão sobre perfeccionismo e psicopatologias.

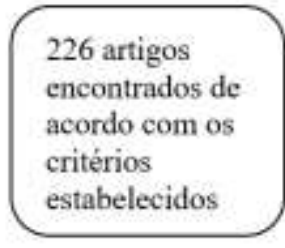

$$
\begin{aligned}
& 13 \text { artigos } \\
& \text { selecionados } \\
& \text { após leitura dos } \\
& \text { textos na } \\
& \text { integra }
\end{aligned}
$$

198 artigos

foram excluidos após leitura critica dos

28 artigos selecionados após leitura critica dos titulos e resumos.

Fonte: Autores. 


\section{Resultados e Discussão}

Abaixo, no Quadro 1, estão dispostos os resultados encontrados no presente manuscrito. Pode-se observar a lista dos 13 artigos que foram indispensáveis para as discussões promovidas. Além disso, foi adicionado dois tópicos que aprofundam as discussões acerca da temática. O primeiro tópico aborda sobre depressão, ansiedade e perfeccionismo. Já o seguindo vai tratar da relação do perfeccionismo com os transtornos alimentares, sobretudo, anorexia e depressão, pois foram expressivamente visualizado nas revisões que compõe o estudo.

Quadro 1 - Título dos artigos, autores e anos.

\begin{tabular}{|c|c|}
\hline Título do artigo & Autor (es) e ano de publicação \\
\hline $\begin{array}{c}\text { Negative Affect and Child Internalizing Symptoms: The Mediating Role } \\
\text { of Perfectionism. }\end{array}$ & $\begin{array}{l}\text { AFRUNTI, N.; \& WOODRUFF- } \\
\text { BORDEN, J.; / } 2016\end{array}$ \\
\hline The Relationship Between Perfectionism and Psychopathology: A Meta-Analysis & LIMBURG, K; et al /2016 \\
\hline Perfectionism and impulsivity based risk profiles in eating disorders & $\begin{array}{l}\text { SOIDLA, K.; \& AKKERMANN, } \\
\text { K. / } 2020\end{array}$ \\
\hline $\begin{array}{l}\text { The interrelationship between orthorexia nervosa, perfectionism, body image and } \\
\text { attachment style }\end{array}$ & $\begin{array}{l}\text { BARNES, M.; \& CALTABIANO, } \\
\text { M. / } 2017\end{array}$ \\
\hline The Association of Rumination and Perfectionism to Social Anxiety & ABDOLLAHI, A. / 2019 \\
\hline $\begin{array}{c}\text { The relationships between perfectionism, anxiety and depression across time in } \\
\text { paediatric eating disorders }\end{array}$ & $\begin{array}{l}\text { MORGAN-LOWES, K.; et al. / } \\
2019\end{array}$ \\
\hline $\begin{array}{c}\text { The problem of overcontrol: Perfectionism, emotional inhibition, and personality } \\
\text { disorders }\end{array}$ & DIMAGGIO, D.; et al / 2018 \\
\hline $\begin{array}{l}\text { How Perfectionism and Eating Disorder Symptoms Contribute to Searching } \\
\text { Weight-Loss Information on the Internet? }\end{array}$ & $\begin{array}{l}\text { PRNJAC, J.; JUKIC, I.; \& } \\
\text { KORAJLIJA, A. } / 2019\end{array}$ \\
\hline $\begin{array}{c}\text { Relationship Between Perfectionism and Depression Among Chinese College } \\
\text { Students With Self-Esteem as a Mediator }\end{array}$ & CHAI, L.; et al. / 2019_ \\
\hline $\begin{array}{l}\text { Perfectionism and eating disorder symptoms in female university students: the } \\
\text { central role of perfectionistic self-presentation }\end{array}$ & STOEBER, J.; et al. (2016) \\
\hline $\begin{array}{l}\text { Social appearance anxiety and dietary restraint as mediators between perfectionism } \\
\text { and binge eating: A six month three wave longitudinal study }\end{array}$ & $\begin{array}{l}\text { BROSOF, L.; \& LEVINSON, C.; / } \\
2016\end{array}$ \\
\hline $\begin{array}{l}\text { Are perfectionism dimensions risk factors for anxiety symptoms? A meta-analysis } \\
\text { of } 11 \text { longitudinal studies }\end{array}$ & SMITH, M.; et al. /2018 \\
\hline Anorexia nervosa and perfectionism: a meta-analysis & $\begin{array}{l}\text { DAHLENBURG, S; ,. GLEAVES, } \\
\text { D. \& HUTCHINSON, A. / } 2019\end{array}$ \\
\hline
\end{tabular}

Fonte: Autors (2021). 


\section{Ansiedade, Depressão e Perfeccionismo}

O perfeccionismo desenvolve um papel crítico nas psicopatologias (Limburg, Watson, Hagger \& Egans., 2017). Embora existam estudos que destoam dessa perspectiva, ao enfatizarem um lado sadio e, consequentemente positivo (Hewitt \& Flett, 1991; Frost et al., 1990 \& Slaney, Rice, Mobley, Trippi \& Ashby, 2001) ainda há uma crescente literatura que enfatiza que o perfeccionismo pode ser desencadear uma variedade de fenômenos psicológicos e patológicos, como por exemplo, depressão, ansiedade, transtorno obsessivo-compulsivo, transtornos alimentares e mentais (Chai, Yang, Jie, Shuyu \& Liu, 2019; Argus \& Thompson, 2008; Ashby, Dickinson, Gnilka \& Noble; 2011; Limburg et al., 2017). Por isso, faz necessário compilar e discutir achados recentes que trazem diversos panoramas sobre esse construto e seus desdobramentos teóricos, empíricos e metodológicos.

Em diversos momentos é comum que as pessoas se sintam ansiosas. Afinal, a ansiedade é uma resposta emocional básica que auxilia a especíe humana a sobreviver e progredir. Com isso, sem a ativação de tal componente frente a um perigo iminente, ficaria dificil de se preparar para fuga ou esquiva diante de um perigo. No entanto, quando a ansiedade é apresentada de forma desreguladora, isto é, perdendo a sua função protetiva, pode-se acionar um sinal de alerta. Frente a isso, sintomas caracterizados por medo persistente e excessivo, preocupação, excitação física e evitação podem demarcar o início de um transtorno patológico (Benson-Martin, Stein, \& Hollander, 2010; Smith et al., 2019).

A depressão é classificada como um transtorno do humor. Pode ser descrita como sentimento de tristeza, perda ou raiva que interferem nas atividades diárias de uma pessoa. Existem diversos fatores que podem desencadear esse transtorno. Alguns deles estão relacionados a história familiar, traumas da primeira infância, uso abusivo de drogas, energia diminuída ou fadiga, dentre outros. Sintomas adicionais favorecem para o desencadeamento da depressão, sendo apetite ou perda ou ganho de peso; insônia ou hipersonia; agitação ou retardo; perda de energia ou fadiga, perda de confiança ou autoestima, pensamentos de suicídio ou tentativa de suicídio. Tais sintomas pode contribuir para o surgimento de psicopatologias (Paykel, 2008)

O perfeccionismo é amplamente descrito como um traço de personalidade que combinam padrões pessoais excessivamente elevados e autoavaliações excessivamente críticas (Hewitt \& Flett, 1991). Dessa forma, o perfeccionismo pode afetar drasticamente os níveis de saúde físico e mental. Ansiedade, depressão e ideação suicida, por exemplo, são apenas alguns dos problemas de saúde mental que os especialistas repetidamente associam a essa forma de perfeccionismo. Sugere-se que devido ao elevado grau de cobrança, o estabelecimento de metas inalcançáveis e o medo costumeiro de falhar e sofrer desaprovação pelas pessoas pode relacionar perfeccionismo e depressão (Black \& Reynolds, 2013).

A esse respeito, uma revisão de meta-análise objetivou testar quais as dimensões do perfeccionismo (se houver) são fatores de risco para desencadear a ansiedade. Para isso, o estudo contou com subsídio de 11 estudos longitudionais com variados públicos (estudantes universitários, amostras clínicas e adolescentes). A partir da exaustiva pesquisa, possivelmente, pode-se relevar que que as dimensões preocupação com os erros, as dúvidas sobre as ações e, em menor medida, os padrões pessoais previam aumentos longitudinais na ansiedade. Em contrapartida, nem o perfeccionismo socialmente prescrito, nem o perfeccionismo auto-orientado, previu mudança em sintomas ansiosos (Smith et al., 2018). De forma adicional, Affrunti \& Borden-Woodruff (2016) apresentam um estudo em que ressaltam a predição do perfeccionismo em relação a ansiedade em crianças e, longitudinalmente, a depressão. De modo específico, é feito uma menção de que o afeto negativo é preditor para as dimensões do perfeccionismo socialmente prescrito e auto-orientado. Essas, por sua vez, podem relevar o aumento de sintomas de ansiedade, depressão e preocupação no público infantil.

Limburg et al. (2017) apresentam investigações sobre o perfeccionismo como uma abordagem transdiagnóstica. Ou seja, o transdiagnóstico são aspectos cognitivos ou comportamentais que contribuem para a manutenção de mais de um distúrbio psicológico (Harvey, Watkins, Mansell, \& Shafran, 2004) e tem sido referido como sendo os pontos de intersecção entre personalidade e psicopatologia (Rodriguez-Seijas, 2015). Adicionalmente, o estudo levantou que as psicopatologias mais 
relativas ao perfeccionismo são transtornos de ansiedade $(6,2 \%)$, seguidos por transtornos alimentares $(4,6 \%)$, Transtornos Obsessivo-Compulsivo (3,9\%) e Depressão (1,6\%). Os sintomas mais avaliados foram com relação a depressão $(28,0 \%)$, seguidos de sintomas ansiosos, transtornos de ansiedade $(19,8 \%)$, TOC $(18,1 \%)$ e, por último, transtornos alimentares $(12,0 \%)$.

Em outra investigação Abdollahi (2019) examinou a associação de ruminação e perfeccionismo à ansiedade social. Ter altos padrões de desempenho considerados inatingíveis, faz com que os perfeccionistas evitem o contato social, por medo de receber críticas, além da crença de que serão desaprovados e que as pessoas esperam nada mais que a perfeição. Por esse motivo, tal relação foi estabelecida uma vez que há os sintomas do Transtorno da Ansiedade Social (TAG) se associam as características do perfeccionismo (e.g. medo de ser avaliado negativamente). A ruminação, é considerada como pensamentos repetitivos, passivos e intrusivos sobre uma determinada situação. Sendo assim, é típico que uma pessoa perfeccionista pense demasiadamente em uma situação para manter seus altos padrões e evitar possíveis ou reais falhas (Nepon, Hewitt, Flett \& Molnar, 2011). O estudo conclui que a ruminação pode contribuir como um mediador para explicar por que e como o perfeccionismo contribui para a ansiedade social.

\section{Perfeccionismo e os Transtornos Alimentares}

O perfeccionismo tem sido alvo de estudos no que se refere aos transtornos alimentares (Brosof \& Levinson, 2017; Farstad, McGeow \& Von Ranson, 2016; Lilenfeld, Wonderlich, Riso, Crosby \& Mitchell, 2006; Soidla \& Akkermann, 2020). Os transtornos alimentares podem trazer impactos graves, inclusive o desenvolvimento de complicações psicológicas, como o aparecimento de depressão e ansiedade (Morgan-Lowes et al., 2019). Especialmente, investigações realizadas com adolescentes têm apoiado a hipótese de que os transtornos estão relacionados a depressão e ansiedade (Holm-Denoma, Hankin, \& Young, 2014)

Há uma vasta literatura que versa sobre os impactos do perfeccionismo na anorexia nervosa (Dimaggio et al., 2018; Norris, Gleaves \& Hutchinson, 2019; Prnjak, Jukic \& Korajlija, 2019 ). Anorexia nervosa (AN) é um distúrbio constituído por distorções cognitivas acerca do próprio corpo. Ou seja, a pessoa com esse tipo de transtorno tem intenso medo de ganhar peso e por isso, faz uso excessivo de atividades físicas e reduz substancialmente a quantidade de ingestão alimentar. Uma metaanalise recente apoiou a hipótese de que o grupo com AN foi mais perfeccionista em comparação com um grupo não clínico. Todavia, o estudo apontou que não houve diferenças significativas no perfeccionismo desadaptativo entre indivíduos com AN e bulimia nervosa (BN) (Dahlenbur, Gleaves \& Hutchinson, 2019).

Além disso, pesquisas sugerem que a dimensão preocupação com erros tem se revelado como um aspecto associado ao perfeccionismo, especificamente, relacionado a um diagnóstico de anorexia e bulimia nervosa (Brosof \& Levinson, 2017; Bulik, Tozzi, Anderson, Mazzeo, Aggen, \& Sullivan 2003). Adicionalmente, é enfatizado que de forma transversal ao longo do tempo, o perfeccionismo pode prever a compulsão alimentar. Ou seja, a ingestão descontrolada de uma quantidade crescente de comida em um curto espaço de tempo, o que pode favorer ao surgimento de comorbidades como por exemplo a anorexia.

Ortorexia nervosa foi introduzido por Steven Bratman em 1997 para descrever um estado envolvendo uma fixação patológica (Bratman, 1977). Apesar de não ter sido reconhecida científica por manuais de diagnóstico, a ON é caracterizada por um interesse em consumir alimentos saudaveis. Dessa forma, indivíduos que são acometidos pela ON eliminam todo e qualquer tipo de alimento que for considerado não-saudável e impuros. Por esse motivo, a literatura tem sugerido que indivíduos com ON também podem ter tendencias de apresentar comportamentos perfeccionistas do tipo consumir uma dieta perfeita e seguir regras de modo rigoroso a fim de não descumprir tal restrição alimentar (Barnes \& Caltabiano, 2016; Koven \& Abry, 2015; Mathieu, 2015) 


\section{Considerações Finais}

O principal objetivo do estudo foi mapear na literatura, por meio de uma revisão integrativa, as principais psicopatologias relacionadas ao perfeccionismo. A personalidade expressa maneiras de agir, pensar, sentir e se comportar no mundo, logo, exercer um desempenho fulcral para a compreensão de psicopatologias e transtornos. A literatura aponta que pessoas com traços tipicamente neuróticas têm mais chances de experimentar sensações desagradáveis, enquanto que no polo oposto, ou seja, baixa pontuação em neuroticismo, as pessoas tendem a apresentar resiliência (Costa \& McCrae, 1992; Soto, 2018).

Ademais, indivíduos que expressam comportamentos conscienciosos podem ser mais propensos a desenvolver traços adaptativos do perfeccionismo. Isso significa que as definições de metas e objetivos aumentam a satisfação pessoal e a confiança, que podem ser vistas como funções psicológicas saudáveis. Já pessoas com pontuação baixa nessa dimensão são desorganizadas, possivelmente não gostam de planejar uma rotina e seguir regras (Soto, 2018)

Esse estudo é potente por trazer uma discussão atual e pertinente sobre o perfeccionismo e os avanços conquistados ao longo de décadas de pesquisas. Com o passar do tempo, esse fenômeno tem sido visto como multidimensional, logo se apresentando como uma natureza adaptativa e desadaptativa. Perfeccionismo desadaptativo refere-se a traços onde um indivíduo tem alto padrões pessoais e é excessivamente autocrítico nas autoavaliações. No contraste, o perfeccionismo adaptativo é considerado um tipo normal e saudávelde perfeccionismo, em que um indivíduo experimenta satisfação por atingir metas que exigem imenso esforço, mas evita ser autocrítico durante contratempos ou falhas (Stoeber \& Otto, 2006; Stoltz \& Ashby, 2007)

Apesar das potencialidades, inúmeras limitações podem ser extraídas desse manuscrito. Em primeiro lugar, consultaram-se três bases de dados nos últimos cincos anos, o que deixa margem para novos estudos ampliarem as bases bem como o recorte temporal de pesquisa. Em segundo lugar, a ênfase se deu em psicopatologias que podem ter o perfeccionismo como um fator favorável. Outras pesquisas poderiam, por exemplo, pesquisar a influência do perfeccionismo em outros contextos, como em atletas de alto rendimento, que são exigidos a atuar com alta performance. Certamente, o nível de autocobrança dos outros e de si pode ser elevado o que seria substancialmente prejudicial à saúde física e mental do atleta. A literatura sobre perfeccionismo embora seja extensa, ainda exige cautela na interpretação e generalização de dados bem como a produção de mais estudos a nível nacional que deem conta de abarcar essa temática que se faz presente e relevante.

\section{Referências}

Abdollahi A. (2019) The Association of Rumination and Perfectionism to Social. Anxiety. Psychiatry. 82(4):345-353.

Afshar, H., Roohafza, H., Sadeghi, M., Saadaty, A., Salehi, M., Motamedi, M., \& Asadollahi, G. (2011). Positive and negative perfectionism and their relationship with anxiety and depression in Iranian school students. Jrms, 16(1), 79-86

Affrunti, N. W., \& Woodruff-Borden, J. (2016). Negative Affect and Child Internalizing Symptoms: The Mediating Role of Perfectionism. Child Psychiatry Hum Dev. 47, 358-368

Andrews, L. M., Bullock-Yowell, E., Dahlen, E. R., \& Nicholson, B. C. (2014). Can perfectionism affect caerer development? Exploring career thoughts and self-efficacy. Journal of Counseling and Development, 92, 270-279. 10.1002/j.1556-6676.2014.00155.x.

Argus, G., \& Thompson, M. (2008). Perceived social problem solving, perfectionism, and mindful awareness in clinical depression: An exploratory study. Cognitive Therapy and Research, 32(6), 745-757.

Ashby, J. S., Dickinson, W. L., Gnilka, P. B., \& Noble, C. L. (2011). Hope as a mediator and moderator of multidimensional perfectionism and depression in middle school students. Journal of Counseling \& Development, 89(2), 131-139.

Barnes, M. A., \& Caltabiano, M. L. (2017) The interrelationship between orthorexia nervosa, perfectionism, body image and attachment style. Eat Weight Disord. 22(1):177-184.

Benson-Martin, J. J., Stein, D. J., \& Hollander, E. (2010). Anxiety disorders. Encyclopedia of Neuroscience, 493-497. Elsevier Ltd.

Bieling, P. J., Israeli, A. L., \& Antony, M. M. (2004). Is perfectionism good, bad, or both? Examining models of the perfectionism construct. Personality \& Individual Differences, 36(6), 1373. 
Bojanić, Ž., Šakan, D., \& Nedeljković, J. (2018). Personality traits as predictors of perfectionism. Facta Universitatis Series Physical Education and Sport, $16(1), 57-71$.

Black, J., \& Reynolds, W. M. (2013). Examining the relationship of perfectionism, depression, and optimism: Testing for mediation and moderation. Personality and Individual Differences, 54(3), 426-431.

Brosof, L. C., \& Levinson, C. A. (2017). Social appearance anxiety and dietary restraint as mediators between perfectionism and binge eating: A six month three wave longitudinal study. Appetite, 108, 335-342.

Bulik, C. M., Tozzi, F., Anderson, C., Mazzeo, S. E., Aggen, S., \& Sullivan, P. F. (2003). The relation between eating disorders and components of perfectionism. The American Journal of Psychiatry, 160, 366-368.

Cerqueira, A. C. D. R.; Cardoso, M. V. L. M. L., Viana, T. R. F.; \& Lopes, M. M. C. O. (2017). Revisão integrativa da literatura: sono em lactentes que frequentam creche. Rev. Bras. Enferm., 71 (2), 453-60.

Chai L., Yang, W., Zhang, J., Chen, S., Hennessy, D. A., \& Liu, Y. (2020). Relationship Between Perfectionism and Depression Among Chinese College Students With Self-Esteem as a Mediator. Omega (Westport). 80(3):490-503.

Costa, P., \& McCrae, R. (1992). Revised NEO Personality Inventory (NEO-PI-R) and NEO Five-Factor Inventory (NEO-FF): Professional manual. Odessa, FL: Psychological Assessment Resources

Dahlenburg, S. C., Gleaves, D. H., \& Hutchinson, A. D. (2019). Anorexia nervosa and perfectionism: A meta-analysis. International Journal of Eating Disorder, 52, 219-229.

Dimaggio G., MacBeth, A., Popolo, R., Salvatore, G., Perrini, F., Raouna, A., Osam, C. S., Buonocore L, Bandiera, A., \& Montano, A. (2018) The problem of overcontrol: Perfectionism, emotional inhibition, and personality disorders. Compr Psychiatry.

Farstad, S. M., McGeown, L M., \& von Ranson, K. M. (2016). Eating disorders and personality, 2004-2016: A systematic review and meta-analysis. Clinical Psychology Review, 46, 91-105.

Flett, G. L., Hewitt, P. L., Shapiro, B., \& Rayman, J. (2001). Perfectionism, beliefs, and adjustment in dating relationships. Current Psychology: Developmental, Learning, Personality, Social, 20(1).

Fowler, S A., Davis, L. L., Both, L. E., \& Best, L. A (2018). Personality and perfectionism as predictors of life satisfaction: The unique contribution of having high school standards for others. Facets, 3, 227-241.

Frost, R. O., Marten, P., Lahart, C., \& Rosenblate, R. (1990). The dimensions of perfectionism. Cognitive Therapy and Research, 14(5), 449-468. Hewitt, P. L., \& Flett, G. L. (1991). Dimensions of perfectionism in unipolar depression. Journal of Abnormal Psychology, 100(1), 98-101.

Frost, R. O., Marten, P., Lahart, C., \& Rosenblate, R. (1990). The dimensions of perfectionism. Cognitive Therapy and Research, 14, 449-468. Hamachek, DE. (1978). Psychodynamics of normal and neurotic perfectionism. Psychology, 15, 27-33.

Haring, M., Hewitt, P. L., \& Flett, G. L. (2003). Perfectionism, coping, and quality of intimate relationships. Journal of Marriage and the Family, 65(1).

Hewitt, P. L., \& Flett, G. L. (1991). Perfectionism in the self and social contexts: conceptualization, assessment, and association with psychopathology. Journal of Personality and Social Psychology, 60, 456

Koven, N. S., \& Abry, A. W (2015) The clinical basis of orthorexia nervosa: emerging perspectives. Neuropsychiat Dis Treat 11:385-394.

Lilenfield, L. R. R., Wonderlich, S., Riso, L., Crosby, R., \& Mitchell, J. (2006). Eating disorders and personality: A methodological and empirical review. Clinical Psychology Review, 26, 299-320

Limburg, K., Watson, H. J., Hagger, M. S., \& Egan, S. J. (2017). The relationship between perfectionism and psychopathology: A meta-analysis. Journal of Clinical Psychology, 73(10), 1301-1326.

Mathieu J (2005) What is orthorexia? J Am Diet Assoc 105(10):1510-1512.

Martin, J. L., \& Ashby, J. S. (2004). Perfectionism and Fear of Intimacy: Implications for Relationships. Family Journal 12(4), 368-374.

Morgan-Lowes, K L, Clarke, P. J. F., Hoiles, K. J., Shu, C. Y., Watson, H. J., Dunlop, P. D., Egan, S. J. (2019) The relationships between perfectionism, anxiety and depression across time in paediatric eating disorders.

Nepon, T., Flett, G. L., Hewitt, P L., \& Molnar, D. S. (2011). Perfectionism, negative social feedback, and interpersonal rumination in depression and social anxiety. Canadian Journal of Behavioural Science/Revue Canadienne Des Sciences Du Comportement, 43(4), 297.

Slaney, R. B., Rice, K G., Mobley, M., Trippi, J., \& Ashby, J. S. (2001). The revised almost perfect scale. Measurement and Evaluation in Counseling and Development, 34(3), 130-145.

Soidla K., \& Akkermann, K. (2020). Perfectionism and impulsivity based risk profiles in eating disorders. Int J Eat Disord. 53(7). 1108-1119.

Paykel, E. S. (2008). Basic concepts of depression. 10(3), 279-289.

Prnjak K., Jukic, I., \& Korajlija, A. L. (2019). How Perfectionism and Eating Disorder Symptoms Contribute to Searching Weight-Loss Information on the Internet? Medicina (Kaunas). 55(10):621. 
Research, Society and Development, v. 10, n. 2, e59410213033, 2021

(CC BY 4.0) | ISSN 2525-3409 | DOI: http://dx.doi.org/10.33448/rsd-v10i2.13033

Smith, M. M, Vidovic, V., Sherry, S. B, Stewart, S. H, \& Saklofske, D. H. (2018). Are perfectionism dimensions risk factors for anxiety symptoms? A metaanalysis of 11 longitudinal studies. Anxiety Stress Coping. 31(1):4-20.

Smith, M. M., Sherry, S. B., Vidovic, V., Saklofske, D. H., Stoeber, J., \& Benoit, A. (2019). Perfectionism and the five-factor modelo f personality: A metaanalytic review. Personality and Social Psychology Review, 23, 267-390.

Stricker, J., Buecker, S., Schneider, M., \& Preckel, F. (2019). Multidimensional Perfectionism and the Big Five Personality Traits: A Meta-analysis.

Soto, C. J. (2018). Big Five personality traits. In M. H. Bornstein, M. E. Arterberry, K. L. Fingerman, \& J. E. Lansford (Eds.), The S AGE encyclopedia of lifespan human development (pp. 240-241). Thousand Oaks, CA: Sage

Stoeber, J. (2018) The psychology of perfectionism: An introduction. In: Stoeber, J. The psychology of perfectionism: Theory, research, applications. Routledge, 3-16.

Stoeber, J., \& Otto, K. (2006). Positive conceptions of perfectionism: Approaches, evidence, challenges. Personality \& Social Psychology Review (Lawrence Erlbaum Associates), 10(4).

Stoeber J., Madigan, D. J., Damian, L. E., Esposito, R. M, \& Lombardo, C. (2017). Perfectionism and eating disorder symptoms in female university students: the central role of perfectionistic self-presentation. Eat Weight Disord. 22(4):641-648.

Stoltz, K., \& Ashby, J. S. (2007). Perfectionism and lifestyle: Personality differences among adaptive perfectionists, maladaptive perfectionists, and nonperfectionists. Journal of Individual Psychology, 63(4). 